\title{
Formación inicial de maestros de inglés en Colombia: Giros y desplazamientos
}

\section{Initial Education of English Language Teachers in Colombia: Turns and displacements}

\begin{abstract}
Pilar Méndez-Rivera
Universidad Distrital Francisco José de Caldas, Bogotá, Colombia

Carmen Guerrero-Nieto

Universidad Distrital Francisco José de Caldas, Bogotá, Colombia

\section{Resumen}

Introducción: La formación inicial docente de maestros de inglés como categoría de análisis comporta una historicidad, que posibilita identificar formas particulares de ver y pensar al maestro de inglés en formación y al inglés como objeto de enseñanza. Objetivo: Explorar las tendencias imperantes en las normas producidas para regular la formación inicial de maestros de inglés y las relaciones de poder-conocimiento que se instalan en los currículos y discursos de formación docente. Metodología: Se utilizaron algunos procedimientos de arqueología foucaultiana para organizar temáticamente algunos discursos dominantes e identificar giros y desplazamientos en la historia de la formación de profesores de inglés en Colombia entre 1990 y 2020. Resultados y Conclusiones: Se produjo un esquema de especificación que marcó dos trayectos equidistantes en las formas de pensar la formación inicial de maestros de inglés: Uno ligado al ideal de la formación profesional, la pedagogía y la investigación y otro, marcado por mecanismos de control y certificación de competencias.
\end{abstract}

Palabras clave: Currículo, desarrollo profesional, formación inicial docente, normas y reforma educativa, maestro de inglés.

\begin{abstract}
Introduction: Initial teacher education as a category of analysis involves historicity what makes it possible to identify particular ways of seeing and thinking of the pre-service English language teacher and English language as teaching object. Objective: Explore the prevailing trends in the norms produced to regulate the initial education of English language teachers and the powerknowledge relationships that are installed in the curricula and teacher training discourses. Methodology: Some Foucaultian archeology procedures were used to thematically organize some dominant discourses and identify displacements and turns in the history of the initial teacher education of English language teachers in Colombia between 1990 and 2020. Results and Conclusions: A specification scheme was produced that marked two equidistant paths in the ways of thinking about initial teacher education. One linked to the ideal of professional education, pedagogy and research, and the other marked by mechanisms of control and certification of competencies.
\end{abstract}

Keywords: Curriculum, educational reform, English teacher, initial teacher education, professional development.
Open Access:

ISSN: $0124-2121$ E-ISSN: $2665-2420$

Editor: Dhayana Fernández Matos TIPOLOGÍA DE ARTÍcULO
Copyright $\odot ~$ By Educación y Humanismo

Correspondencia: pmendez@udistrital.edu.co

Recibido: 30-09-2021 Aceptado: 15-12-2021 En línea desde: 19-01-2022

Cómo citar este artículo (APA): Méndez-Rivera, P., \& Guerrero-Nieto, C. (2022). Formación inicial de maestros de inglés en Colombia: Giros y desplazamientos. Educación y Humanismo, 24(42), 68-89. https://doi.org/10.17081/eduhum.24.42.4662 


\section{Introducción}

La formación inicial de profesores se plantea como una fase clave del desarrollo profesional docente (Vaillant, 2010, Tenti, 2009; Terigi 2009, entre otros) pero también de control social (Popkewitz, 1990; Giroux, 1987) para el aseguramiento de la calidad y el funcionamiento del sistema educativo. Se espera que, en esta fase, las instituciones formadoras de maestros garanticen a la sociedad que la formación del futuro maestro sea robusta en conocimientos, competencias, destrezas y habilidades para enseñar la disciplina de su especialidad y al mismo tiempo responder a los constantes y cambiantes desafíos que se le presenten. Esto implica no sólo abordar las relaciones teoría-práctica, pensamientoacción (Davini, 1995), disciplina-pedagogía, reflexión-evaluación, sino también los enfoques de formación docente y la flexibilidad de sus currículos para adaptarse a las expectativas específicas de formación de un momento determinado.

En Colombia, las finalidades de la formación de educadores, sancionadas en la Ley General de Educación en 1994, fueron determinantes para establecer un marco de comprensión de la formación inicial docente cimentado en los discursos de profesionalización de la docencia para asegurar el reconocimiento de la pedagogía y la investigación en el perfil de formación de los educadores. En la ley, elementos como la calidad e idoneidad ética y pedagógica del futuro educador hicieron posible que los programas de formación inicial resignificaran la práctica y teoría pedagógicas desde un componente de formación investigativa vinculado a las disciplinas específicas para la obtención del título como licenciado. Sin embargo, a la fecha, muchas han sido las modificaciones introducidas a la Ley General de Educación, algunas de las cuales son materia de análisis en este documento.

Las leyes 30 de Educación Superior (1992) y 115 General de Educación (1994), introducen un nuevo dominio: "velar por la calidad de la educación/servicio educativo", lo que generó todo un sistema de aseguramiento de la calidad de la educación superior. En consecuencia, la formación inicial docente debe cumplir con los criterios de calidad establecidos por un andamiaje de calificación y acreditación para el otorgamiento del registro calificado de las licenciaturas (Calvo, 2004; Jurado, 2016). La Acreditación de Alta Calidad surge entonces, como el mecanismo que actualiza las distintas reformas a las que las instituciones formadoras de docentes se han visto abocadas para poder seguir funcionando.

La evolución de la formación inicial de los maestros está vinculada a la historia de la educación y sociedad colombianas, y a las políticas educativas que demandan reformas a los programas de formación inicial docente para la consolidación de competencias, metas e indicadores. Este escenario no está exento de tensiones y contradicciones frente a la limitada autonomía de dichos programas, que juegan también a constituirse en procesos de asimilación, apropiación, pero también de resistencias (Martínez, et al., 2015) a las leyes y 
discursos que le instalan en lo meramente técnico y operativo, coartando su accionar crítico y político.

La formación inicial de profesores de inglés no escapa de este razonamiento que conmina su obediencia a las disposiciones normativas, a la vez que va desarrollando esquemas de resistencia creativa a algunas de las demandas de ley, razonamiento experto o naturalizaciones sociales. Aunque son escasos los estudios que han vinculado históricamente la formación inicial de maestros de inglés en Colombia en relación con el eje normativo, se destacan los realizados por Cárdenas (2009), Cárdenas (2010), Castañeda, (2019), Fandiño-Parra (2011, 2013), González \& Álvarez (2010) y Granados, (2015). Una revisión de estos trabajos permite colegir ciertas tensiones frente a la necesidad de formar un maestro más comprometido con la educación colombiana, su contexto y su capacidad agente para resolver problemas y proponer alternativas pedagógicas (Castañeda, 2019).

Como corolario de estas revisiones, este artículo de investigación intentó responder la pregunta: ¿Cuáles han sido las transformaciones introducidas en la formación inicial de maestros de inglés a partir del entramado normativo? Los resultados de esta indagación hacen posible identificar algunos giros y desplazamientos en la formación inicial de maestros de inglés en Colombia en clave de su implementación en programas de formación docente.

La formación inicial de profesores es depositaria de las expectativas de formación para responder a las demandas políticas, sociales, culturales y educativas de la sociedad. La fase inicial de la formación docente es la encargada de suministrar las bases que permitan al profesor en formación construir el conocimiento pedagógico especializado, los principios y las reglas prácticas para poder afrontar todo tipo de tareas y problemas (Elbaz, 1983). Diversas lecturas de las normas y prácticas que intervienen en la formación docente ponen de manifiesto el poder político y simbólico que se articula en los discursos del deber ser como configuraciones idealizadas de identidad del futuro docente que se construyen con los idearios de quiénes ostentan el poder y guían los imaginarios sociales, los cuales, vía normalizaciones y prescripciones (Munévar-García, 2019), regulan aspectos puntuales de la formación (v.g. relación pedagogía y disciplina), su diseño curricular (v.g. teoría-práctica, interdisciplinariedad, competencias) y la proyección del perfil de formación del futuro educador (v.g. investigador, reflexivo, crítico, emancipador, analista, entre otros).

Los diagnósticos en materia de formación inicial del profesorado insisten en identificar esta fase como decisiva para lograr los cambios requeridos en el docente, para que, a su vez, la escuela cambie. La modificación del currículo sirve entonces para priorizar conocimientos relevantes y especializados que permitan la consolidación del conocimiento profesional base (Perrenoud, 2001; Imbernón, 2001; Shulman, 1987). Para los maestros de inglés, dicho conocimiento informa tres grandes áreas:

i. El contenido de L2 de los programas de formación docente,

ii. Las pedagogías para enseñar la $L 2, y$ 
iii. Las formas institucionalizadas para aprender a enseñar contenido y pedagogía (Johnson, 2009).

La propuesta de Shulman (1987) para entender el conocimiento base de la enseñanza, en relación con un correlato de enseñanza efectiva, reconoce los saberes pedagógicos y disciplinar, como elementos consustanciales para garantizar que el futuro maestro pueda pensar y ejecutar la enseñanza de la disciplina de su especialidad.

Cuando se revisan las tendencias de formación imperantes en la literatura angloamericana y se contrastan con las reportadas en algunos programas de formación docente en lenguas extranjeras en el país, se identifican tres tendencias que se suceden o solapan de acuerdo al énfasis de formación: "teacher training (concebida como entrenamiento y formación de competencias específicas), teacher education (profesor reflexivo); y teacher development (proceso autoformativo y de desarrollo profesional)" (Madrid, 2004, p.2).

Lo anterior pone de manifiesto la tensión que la relación entre lo pedagógico y lo disciplinar ha constituido para los currículos de formación inicial para superar la noción de entrenamiento y balancear las asignaturas dedicadas a la adquisición del inglés por parte de los futuros maestros y las asignaturas dedicadas a su formación pedagógica. Sin embargo, la mediación entre lo disciplinar (la lingüística aplicada) y lo pedagógico (la enseñanza de la lengua inglesa) no se resuelve con los contenidos de la formación per se. La pedagogía tampoco puede reducirse simplemente a teorías y estrategias o confundirse con la didáctica, ni mucho menos reducir la disciplina a la asignatura o al contenido (Flórez, 1997; Saldarriaga, 2009; Tezanos, 1982). Pero, si algo ha sido determinante en las normas y prescripciones que regulan la formación de docente de inglés en Latinoamérica ha sido el paradigma del hablante nativo ideal para medir y evaluar el aprendizaje del inglés.

Ahora bien, la relación teoría-práctica es una tensión constante que problematiza la buena formación docente. Distintas voces (Vasco y Martínez-Boom, 2008; Zuluaga, 1999) reconocen que "el saber en la práctica trasciende el mero hacer las cosas" (Zuluaga, 1999, p.26) y comporta el conocimiento derivado de la experiencia vivida y adquirida en estos espacios de aula y escuela, en interrelación con los procesos meditados y reflexionados de la teoría aprendida. La enseñanza (del inglés) como práctica comporta una complejidad en sí misma, pues no sólo se trata de aplicar teorías para dar la lección, sino que la lección se orienta a enseñar una lengua extranjera; esto supone la operativización de un conjunto de acciones que tienen lugar antes (planning) durante (action) y después de la práctica (evaluation) (Richards \& Lockhart, 1994).

La experiencia vivida en la escuela y el aula posibilita la adquisición de otros tipos de conocimiento que trascienden el conocimiento disciplinar (Martínez-Boom, 2016). El lugar que ocupan los espacios de práctica en las mallas curriculares busca explorar esta especificidad en la enseñanza, así como las habilidades del docente en formación para la 
hacer las negociaciones entre la teoría y la práctica. Este horizonte aquí descrito permite señalar que la formación inicial docente es una fase atravesada por exigencias y tensiones que la desafían permanentemente a la luz de los cambios introducidos al sistema de educación superior que regula esta fase de formación.

\section{Método}

El análisis histórico del discurso desde la arqueología Foucaltiana (Foucault,1969) ofrece unos procedimientos para la identificación de formaciones discursivas y sus relaciones con otros enunciados, tales como la eventualización, la tematización y la formación de objetos y modalidades enunciativas. Este ejercicio hace posible rastrear discursos de poder-saber en torno a la formación inicial de maestros e identificar aquellos que histórica y temáticamente son dominantes. El archivo construido permitió señalar regularidades y giros importantes en la formación inicial de maestros de inglés en el país.

\section{Corpus}

El corpus estuvo constituido por leyes, decretos, planes y resoluciones (Ver anexo 1), lo que permitió la construcción de un archivo temático que articula dos elementos: este eje normativo y la producción académica de formadores de maestros en el área. Para la construcción de dicho archivo se parte de:

- Identificación de fuentes documentales y lectura temática de los registros: Búsqueda y rastreo histórico de los documentos del corpus; cruce de los mismos para identificar temas comunes.

- Lectura crítica de los registros: Se realiza una rejilla de especificación que permite visualizar históricamente eventos con formaciones discursivas recurrentes.

- Identificación de enunciados, funciones enunciativas y condiciones de funcionamiento: Se describen regularidades, discontinuidades, contradicciones y actualizaciones

\section{Procedimientos}

El análisis del archivo obligó la desarticulación de la presunta unidad del texto para establecer relaciones con otros textos y discursos que permiten identificar temas recurrentes, advertir la continuidad o la discontinuidad en una regularidad discursiva. También posibilitó trazar una línea de tiempo que sirve para ilustrar lo discontinuo y la emergencia de nuevas formas de comprender la formación inicial. Los resultados de este análisis permitieron identificar temas como superficies de emergencia con cierta regularidad y continuidad para denotar un giro con claras irrupciones enunciativas (formación 
investigativa, formación permanente) pero también desplazamientos que sujetan a los maestros en formación y a los programas de formación docente (caso del desplazamiento hacia la competencia certificada).

\section{Resultados y Discusión}

En este apartado se organiza temáticamente la relación reforma -formación inicial docente para nominar e identificar giros y desplazamientos que posibiliten entender las prácticas de gobierno que operan en las distintas transformaciones introducidas históricamente a la formación inicial de maestros de inglés. Luego de presentar los temas, sus giros y desplazamientos, se introduce un esquema de especificación (figura 1) con el objetivo de señalar la discontinuidad en la forma de comprender la formación inicial de maestros de inglés, discutiendo dos trayectos equidistantes.

\section{El ideal de la formación docente}

La década del 90, como ninguna otra en Colombia, registró la emergencia de varias disposiciones normativas (leyes, decretos, planes) alrededor de la educación. Estas pretendían dar bases legales a una serie de discursos y prácticas, que venían gestándose en distintos estamentos nacionales e internacionales (v.g. Movimiento pedagógico colombiano, UNESCO, 1990; CEPAL, 1992, MEN, 1995), y que reclamaban un cambio, en el cual era decisivo el papel profesional del personal docente. Los considerandos legislativos atribuían a los modelos técnicos, conductistas y trasmisionistas de formación docente la falta de un maestro capaz de enseñar a sus estudiantes a aprender y, que, en consecuencia, coadyuvara al cambio de prácticas de enseñanza memorística y transmisionista propias de las tradiciones práctico-artesanal y técnico-eficientista observables en las clases de los maestros (CAENS, 2000, UNESCO, 1991). Sin embargo, habría que notar que las luchas de los maestros colombianos fueron decisivas para detonar cambios mucho más profundos en la consideración social y profesional de sus saberes y prácticas, irradiando un dispositivo de relaciones instituyentes con la pedagogía, la investigación y la cultura más allá de los dictámenes de ley.

En todo caso el ideal de la formación docente como enunciado reclamaba un educador que cumpliera con las funciones profesionales e investigativas y de servicio social del encargo, con la capacidad de promover innovaciones, aprendizaje cooperativo y autónomo:

Ha de ser un profesional de la educación, capaz de producir conocimientos e innovaciones en el campo educativo y pedagógico; de superar ; de garantizar que los educandos se apropien del mejor saber disponible en la sociedad y de crear condiciones agradables en la institución educativa para el autoestudio y el auto aprendizaje grupal cooperativo (...), que el educador sea capaz de lograr que la apropiación de los conocimientos ocurra en un ambiente democrático, de autoestima y solidaridad; y, que 
las mejores experiencias y conocimientos pedagógicos construidos sean sistematizados, acumulados y reproducidos por las siguientes generaciones de profesionales de la educación, lo cual significa formar tradición pedagógica (Ministerio de Educación Nacional, 1996).

Así, los discursos en torno a la formación docente, dispersos en una serie de disposiciones normativas, destinadas a desarrollar los preceptos de las leyes 115 de 1994 y Ley 30 de 1992, articulaban la idea del docente como un profesional de la educación que actuara con profesionalismo y se asumiera a sí mismo como profesional e intelectual. Vale la pena señalar que las aspiraciones por la profesionalización de la docencia se remontan a la década del 70, época en la que los maestros colombianos, organizados en sindicatos, lucharon por la dignificación de la carrera docente y la expedición de un Estatuto Docente (Méndez, 2015).

Posteriormente, las luchas de los maestros produjeron en Colombia, un movimiento pedagógico que reclamaba como condiciones constitutivas del maestro su relación con la pedagogía, la investigación y la cultura. Se esperaba entonces, que, el maestro derivara de sus prácticas de enseñanza, estrechas relaciones con la investigación y la producción de conocimiento pedagógico y disciplinar, aspiraciones que el Movimiento Pedagógico Colombiano ligaba a la condición de maestro. Aunque no se encontraron vínculos directos entre el área de Enseñanza del inglés (ELT por sus siglas en inglés) con el Movimiento Pedagógico, sí pueden notarse una serie de acciones y prácticas de formación, investigación y especialización posgradual dentro y fuera del país, así como también la emergencia de revistas especializadas, y maestrías (finales del 90, principios de la década del 2000) que generaron nexos vinculantes con las subjetividades emergentes de formadores de docentes investigadores, autores, editores, así como a los docentes en formación como destinatarios y lectores del conocimiento y reflexiones situadas en el campo de la enseñanza del inglés.

\section{El giro hacia la formación investigativa y a la especificidad}

Los programas de formación inicial de profesores fortalecieron su discurso de las profesiones en los correlatos de profesionalidad y profesionalismo para ofrecer una formación que atrajera a aspirantes dispuestos a formarse con este espíritu. El giro hacia la formación investigativa, el saber pedagógico y los énfasis en saberes específicos (MEN. Decreto 0272 de 1998) se convirtió desde entonces en formaciones discursivas constituyentes de las misiones de formación de los programas de licenciatura colombianos y de sus nuevas denominaciones. La interpretación del decreto para algunos programas de licenciatura de lenguas significó ganar en el terreno de la especialización, lo cual derivó en la necesidad de proponer esquemas interdisciplinarios para no sacrificar la mirada compleja de la formación docente, pero se perdió en el perfil ocupacional, pues para algunas licenciaturas en lenguas modernas (español-inglés-francés), filología e idiomas, entre otras, que habilitaban la enseñanza del español con la titulación, tuvieron que ceder este campo de actuación laboral a las licenciaturas en lengua castellana con la aplicación el decreto 0272 
de 1998.

En el plano de la especialización, los efectos para los planes de estudio y los currículos fueron inmediatos por el incremento de asignaturas orientadas a la lengua extranjera y los acentos en los acometidos misionales en el período subsecuente (2001-2010) marcados en la formación de docentes investigadores, reflexivos (CAENS, 2000), competentes, con alto dominio de una o dos lenguas extranjeras, analistas, entre otros (Una revisión de diez licenciaturas en el país permite colegir dicha información).

Vale la pena aclarar que algunas de estas apuestas habrían sido el referente de un proyecto marco denominado Colombian Framework for English (COFE) entre 1991-1997, cuyas acciones estaban orientadas al mejoramiento de la enseñanza del inglés en Colombia en varios ámbitos: el desarrollo de la lengua, la formación profesional, la evaluación y los recursos (Rubiano, Frodden y Cardona, 2000). También se debe destacar el papel de los programas de formación posgradual que asumieron el encargo social de formar docentes investigadores a través de la promoción de la investigación cualitativa en educación con el espíritu de cercar la brecha entre la práctica reflexiva y las prácticas investigativas (Pineda \& Clavijo, 2003). Esto, permite advertir desplazamientos importantes en el entendimiento de la formación docente de transmisionista a transformadora (Freire, 2011; Cummins, 2011) a saber:

- Cierto consenso frente al papel del formador de maestros en lograr la intersección entre lo pedagógico y lo disciplinar y en discutir estas tensiones: teoría/práctica, investigación/acción, dominio de lengua/enseñanza efectiva.

- La necesidad de relaciones más evidentes entre la teoría y la práctica que trasciendan el mero dictar la clase para la formación de un profesor crítico y reflexivo capaz de evaluar y transformar su propia práctica.

- La importancia de la reflexión, la crítica y la evaluación para la construcción del saber pedagógico que se adquiere en la práctica y el status que este saber merece.

- El papel protagónico del futuro profesor para consolidarse como hablante competente de la lengua extranjera en todas sus habilidades.

- La importancia de la reflexión, las experiencias previas de formación e historia escolar en la formación del futuro docente.

- La necesidad de actualización permanente durante y después de la titulación como garante de la calidad en el ejercicio de la docencia.

- La ubicación estratégica de la práctica docente en los planes de estudio para articular las apuestas específicas de formación. 
Una vez focalizada la especialización en la disciplina, la lingüística aplicada a la enseñanza del inglés y la pedagogía de la lengua extranjera, un conjunto de saberes disciplinares irradió los planes de estudio e intensificó las lenguas extranjeras a dominar. La preocupación por el dominio de la lengua condujo a los programas de licenciatura en Inglés a tener, por un lado, como principales fuentes de consulta, citación e inspiración, al conocimiento producido en países angloparlantes para la organización de los proyectos educativos institucionales; y del otro, el énfasis dado al inglés como lengua de instrucción y no al español-inglés como correlato de bilingüismo, máxime cuando existen voces que reclaman el reconocimiento de esta última práctica como válida y necesaria (De Mejía, 2006; Guerrero, 2008; 2009; Valencia, 2005; Cárdenas-Vergaño, 2018).

Los programas de formación docente tenían la potestad de introducir en sus proyectos educativos el andamiaje conceptual, teórico, metodológico necesario para fundamentar sus propuestas de formación y definir, entre otros tantos aspectos, perfiles de formación, modelos pedagógicos, el estatuto de la práctica, el estatuto de la teoría, el tipo de relaciones establecidas con la pedagogía, la didáctica, la investigación. Como corolario de esta empresa, la singularidad de un ethos servía para consolidar un distintivo que, coloquialmente se traducía en un sello que distinguía a los egresados de $x$ o $y$ universidad y programa.

\section{El giro hacia el desarrollo profesional docente}

El desarrollo profesional docente ligado a la profesionalización de la docencia en Colombia (Decreto 1278 de 2002) vinculó el rendimiento y el crecimiento profesional como actividades continuas y necesarias para el ejercicio de la docencia (Cabra \& Marín, 2015). En tal sentido, el esquema de actualización pedagógica y de conocimientos específicos moviliza una serie de acciones de capacitación docente paralelas y/o simultáneas durante la carrera y después de la titulación mediante programas de formación posgradual, cursos de perfeccionamiento docente, congresos, seminarios, diplomados y eventos certificados.

La formación profesional extendida a lo largo de la vida (Ávalos, 2014), para que los docentes sean capaces de responder a las cambiantes condiciones del contexto (Vaillant, 2009, Teregi, 2009), fue acogida y respaldada por discursos de saber experto y académicos locales que atribuían a estas prácticas de actualización y formación continua y permanente (Cuenca, 2005; Nieva \& Martínez, 2016) un impacto positivo en el conocimiento y el desarrollo profesional docente. De hecho, un estudio realizado por González (2003), en el área de formación de profesores de lenguas, exploró las necesidades de capacitación de los profesores de inglés. Se encontró que éstos reclamaban oportunidades de capacitación menos formales y estructuradas como los congresos del área y las charlas de las casas editoriales como generadoras de actualización en los perfiles docentes como trabajadores, instructores y aprendices. Las preguntas alrededor de las características ideales de un 
programa de formación de docentes de inglés y del impacto de los programas de desarrollo profesional para el logro de metas de los formadores de docentes (González \& Quinchía, 2003) empezaban a perfilar un conjunto de posibilidades de desarrollo para el formador de educadores. Todas ellas ligadas a la investigación, el contexto y la enseñanza, tales como conocimiento de las realidades locales, amplia experiencia en la enseñanza del inglés, dominio de la lengua y experiencia en investigación.

En consecuencia, se reclamaban acciones institucionales e individuales más contundentes para enfrentar los retos y necesidades de desarrollo profesional docente (González \& Sierra, 2005). Se requería que involucrasen la participación en comunidades de profesores, trabajo colaborativo en red, una enseñanza reflexiva (Jerez, 2008) y la participación como ponentes en conferencias nacionales e internacionales como parte de una agenda de desarrollo profesional.

El archivo de la producción académica del área de ELT (2000-2016) permite precisar que las fuentes de inspiración, consulta y citación de los artículos dedicados al tema de la formación, desarrollo profesional y conocimiento del profesor de lengua (Macíaz, 2013) eran tomadas de la producción de conocimiento de autores tales como Freeman, 1991; Nunan 1991; 1994; Shulman, 1987; y Wallace 1991) entre otros. Lo anterior destaca un desplazamiento geográficamente localizado para la citación que favorece los conocimientos del norte global producidos en inglés en comparación con la citación mucho más escasa (2010-2017) de trabajos realizados en el Sur global. Las revistas en ELT, a inicios de la década del 2000, declaraban como conato de su misión, promover los conocimientos de las realidades locales y algunos trabajos inspirados en Kumaravadivelu, 1994, 2002 (González, \& Quinchía, 2003) y no en los trabajos de pedagogos o intelectuales en Colombiareclamaban la construcción de una pedagogía post-método.

Ahora bien, de la mano del desarrollo de las revistas especializadas de mayor trayectoria (Colombian Applied Linguistics Journal, Profile, Ikala, How), se destaca también la emergencia del autor/autora en la subjetividad del formador de maestros en clave de su propio desarrollo profesional y de líneas de investigación con potencia para irradiar relaciones con otros autores.

\section{El desplazamiento hacia la competencia certificada}

La década del 2000 marcó episodios críticos que desplazaron la manera como se había vislumbrado el ideal de la formación. En principio, el Estatuto Docente 1278 de 2002 introduce un desplazamiento identitario que define como profesionales de la educación ya no sólo a los licenciados en educación y normalistas superiores, sino también a los profesionales con título diferente "habilitados para ejercer la función docente", sin distingo o reparo alguno de la especificidad de la preparación profesional para el ejercicio de la enseñanza. En consecuencia, "surge una ruptura epistemológica en la forma de pensar al sujeto de la enseñanza que desplaza el valor concedido al ideal de la formación específica 
en pedagogía, y le relega a un nivel secundario, de tipo complementario", dándole mayor cabida a la definición de la función docente (Méndez, 2018, pp.318-320).

En clave de políticas lingüísticas para el país, el lanzamiento del programa Bogotá Bilingüe en el año 2004 detonó una serie de discursos y prácticas en torno al interés por el dominio del inglés. El impacto de este programa derivó en un conjunto de objetivaciones para pensar al maestro de inglés como deficitario de competencias lingüísticas en comparación con las de los "hablantes nativos del inglés" y, en consecuencia, la necesidad de elevar su nivel de lengua y demostrarlo vía certificación (Acuerdo 253 de 2006). Distintos proyectos, planes y programas (Fortalecimiento de una segunda lengua, Plan Sectorial de Educación 2004-2008-Bogotá una gran escuela, Plan Decenal de Educación 2006-2016, Plan Sectorial de Educación 2008-2012, Estudio Teach Challenge, 2008-2013, Programa Nacional de Bilingüismo 2004-2019, Programa de Fortalecimiento al desarrollo de competencias en lenguas extranjeras, 2010-2014 y recientemente, el Plan Nacional de Inglés 2015-2025 en Colombia) han ido articulando la necesidad de la certificación del nivel de lengua como requisito indispensable para medir/garantizar el dominio del inglés de docentes y estudiantes. Los efectos de este nuevo enclave certificación/garantía fraguaron una serie de prácticas que ameritan ser discutidas:

1. La certificación para diferenciar la calidad del empleo y del salario para la contratación de profesores en el país, con mayor impacto en el sector privado que en el sector público.

2. La certificación por encima del título de licenciado que marca un nuevo acento en la despedagogización de la profesión docente en el área.

3. La certificación para la consideración social del profesor de inglés como competente o no competente.

Dicho de otro modo, de una noción de licenciado (pedagogo) o profesional de la lengua extranjera a hablante certificado en B2/C1 (Niveles de competencia altos en el Marco Común Europeo) operan una serie de reduccionismos que centran la mirada primordialmente en el dominio de la lengua inglesa y no en los conocimientos para enseñarla, entre otros componentes de la formación docente (axiológico, político, social, cultural, etc.).

Este desplazamiento en la manera de pensar al maestro de inglés ha conllevado a que se sospeche del diploma que lo acredita como licenciado para garantizar el dominio de la lengua Inglés. De hecho, la más reciente reforma (Colombia, Res. 2041 de febrero de 2016) ha exigido a las licenciaturas encargadas de la formación del profesor de inglés, que incluya la certificación de un nivel $\mathrm{C} 1$ como requisito de su titulación. Para ello determinó las pruebas Saber Pro o el conjunto de exámenes de agencias internacionales con validez para el Ministerio de Educación Nacional. Es decir, vía reforma se instala un nuevo mecanismo de control y regulación propio de una práctica de certificación para el trabajo. 
Como consecuencia, algunas licenciaturas han resistido por mucho tiempo esta práctica de la certificación por considerarla inserta en una noción de mercado que favorece la racialización de la identidad docente (Castañeda-Peña, 2018; Rosa \& Flores, 2015; Kubota, 2014) y desconoce las prácticas locales de formación. De hecho, en este último período 2006-2015 se registra el rechazo a la imagen del profesor de inglés como mero instructor de lengua $y$, la consolidación de una nueva regularidad discursiva que reclama la subjetividad de "educador" para el maestro de inglés que, otrora (década del 80) había sido reclamada para los maestros por el Movimiento Pedagógico.

De hecho, como una manera de reconocer los saberes constituyentes de la formación específica de estas licenciaturas, algunos programas han optado por otros mecanismos de comprobación de la idoneidad de sus estudiantes, a través de la promoción de la escritura y defensa de los trabajos de grado, la escritura de artículos, y participación en ponencias en lengua inglesa, entre otras actividades que, no sólo enfatizan el dominio de la lengua sino competencias pedagógicas, didácticas, e investigativas más ligadas con la academia, la investigación y la reflexión, aspectos estos que no se miden en los exámenes de competencia de lengua.

También, se producen en la literatura especializada enunciados como global/local, glocal para rescatar/conciliar pedagogías emergentes en las que se haga evidente el rol del educador como productor de saber localmente situado. Por consiguiente, llamamos la atención sobre las apuestas contemporáneas de líneas de investigación, aún a pesar de las intervenciones normativas vía acreditación, de programas de formación inicial y pos gradual. Estas han circunscrito su quehacer en las epistemologías del sur en procura de descolonizar el ser, el saber y el poder con la aspiración de identificar situaciones coloniales e irradiar cambios en el modelo de formación docente imperante imbuido en el enclave de la certificación (en la última reforma a las licenciaturas).

Finalmente, aspectos de sensible intervención pueden notarse en el Artículo 222 de la Ley 1753 de 2015/Plan Nacional de Desarrollo, pues cambió el carácter voluntario de la acreditación al imperativo de obligatorio, concediendo términos perentorios para su acreditación so pena de la pérdida del registro calificado para el funcionamiento de las licenciaturas. Esto significa una violación flagrante a la autonomía de las universidades y un desconocimiento de los propios procesos de autoevaluación y de las tareas concretas de plan de mejoramiento vinculadas a presupuesto. Sumado a ello, la Resolución 2041 de 2016 ordenó a las licenciaturas a cambiar sus propias denominaciones y optar por las opciones propuestas en la misma resolución que también definió parcialmente las competencias del educador y contenidos curriculares.

Ante la obligatoriedad de ley, las licenciaturas -incluidas las ya acreditadas- cambiaron su denominación y organizaron sus planes de estudio tratando de armonizar lo que la norma prescribía con lo que consideraban sus pilares identitarios, entre estos aspectos, el componente práctico normado en 50 créditos, lo que implicó para muchos programas 
proponer alternativas creadoras de la relación teoría-práctica. En otras palabras, acreditación/certificación como prácticas de regulación atraviesan no sólo a los sujetos sino también a las instituciones, objetos y conceptos de la formación profesional.

\section{Trayectos de la formación inicial de maestros de inglés}

Como puede colegirse por los temas resultados del análisis, la formación inicial docente en el campo del ELT ha sido atravesada por una serie de reformas en las que se tejen prácticas de subjetivación, algunas interpelan a los sujetos a aceptar los roles configurados y preestablecidos en ' $x$ ' 0 ' $y$ ' posición de sujeto, pero otras son rechazadas y activan la resistencia y capacidad creativa para reacomodar lo preestablecido (Foucault, 1996). El esquema de especificación (figura 1) que se presenta a continuación permite identificar 2 trayectos importantes en las formas de pensar la formación docente.

Un primer trayecto caracterizado por la elevación del estatuto profesional del docente en el que la formación profesional estaba ligada a la idoneidad pedagógica, la investigación y el conocimiento de saberes específicos. El papel de las licenciaturas se consideraba crucial para cumplir con el encargo de formar maestros comprometidos con las agendas derivadas de la docencia y la investigación. La manera como se aceptó el giro hacia el desarrollo profesional docente (González y Sierra, 2005; Mora, Trejo y Roux, 2014) fue clave para el desarrollo de programas de formación posgradual, cursos, talleres y eventos en el territorio colombiano. Estos brindaron las condiciones para que los maestros articularan prácticas de estudio, escritura, autoformación, que derivaron en un sinnúmero de perfiles constituyentes tales como: investigador, autor, ponente, tallerista, consultor, experto en evaluación, gestor de proyectos, gestor académico, editor, educador.

Un segundo trayecto caracterizado por la validación/evaluación de competencias en el que el desplazamiento hacia la certificación implica para los programas de formación docente una gestión añadida para acreditar el cumplimiento del nivel de lengua de los profesores. Este desplazamiento, inserto en la subjetivación del nativo, impactó la consideración social del maestro de inglés, en adelante sometido a un esquema de clasificación para aspirar a la nivelación C1/C2 y entrar en abierta competencia con los profesores extranjeros nativos o certificados.

También impacta las prácticas de gobierno de las licenciaturas, que pasan de una acreditación voluntaria a una acreditación obligatoria para su funcionamiento (Artículo 222 de la Ley 1753 de 2015/Plan Nacional de Desarrollo), sumando a sus prácticas de formación y titulación el aprendizaje de una "lengua extranjera por decreto" (El Espectador, 27 de abril de 2017), que obliga certificaciones en el nivel C1 para los licenciados en lenguas extranjeras. En consecuencia, la sombra de la sospecha se cierne sobre la calidad de la formación profesional de las licenciaturas y conmina a estas a resistir creativamente ante esta lógica de mercado. Los licenciados deberán demostrar que su formación/titulación 
como pedagogos de una lengua extranjera es determinante para superar el reduccionismo que supone la valoración de su formación desde una perspectiva de manejo de lengua.

Figura 1.

Esquema de especificación: trayectos en la formación docente

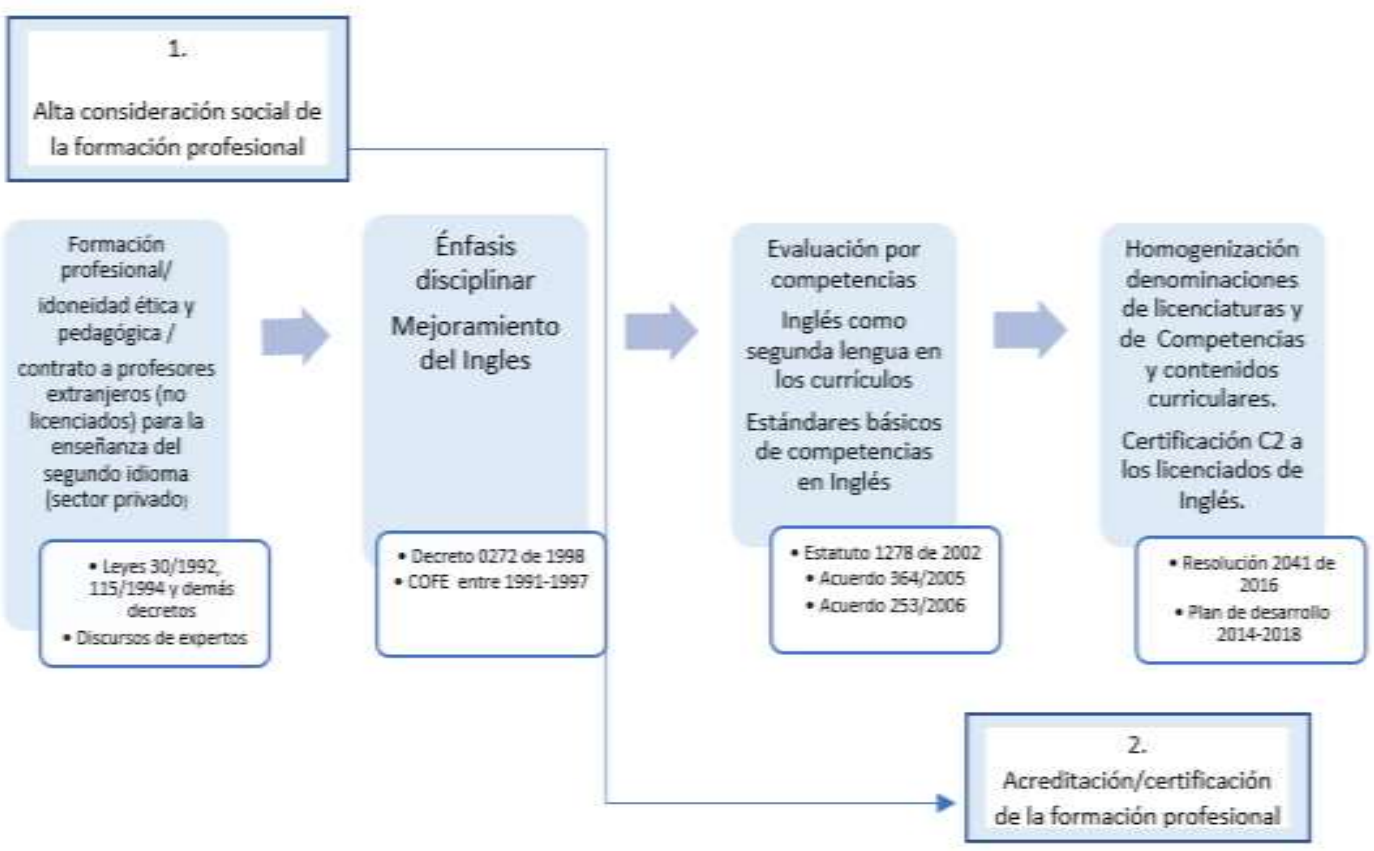

Fuente: elaboración propia (2021)

Este segundo trayecto pone de manifiesto la insistencia de la norma por la homogenización, lo cual comporta un claro intento por romper con las prácticas y discursos identitarios de los programas de formación que, en la búsqueda de su singularidad e diferencia, habían logrado distinguirse por su innovación curricular, modelo pedagógico, y sello académico para formar profesionales al amparo de sus principios fundantes. Del mismo modo, se marca una notoria contradicción con la regularidad alrededor de la calidad que se había negociado con las licenciaturas ya acreditadas, desconociendo sus condiciones de alta calidad y reposicionándolas como sujeto de reforma.

Por último, el desplazamiento (en comparación con el primer trayecto) permite colegir que no existe claridad en torno a un ideal de formación que dibuje contornos definidos del tipo de sujeto que se quiere formar. Se exigen tantos saberes, funciones y tareas al futuro maestro (para que sepa hacer de todo un poco) y, al mismo tiempo, vía reforma, se exacerban la multiplicidad de exigencias a las licenciaturas, que resulta evidente la violencia jurídica que se ejerce contra las tradiciones, las experiencias y las trayectorias de las licenciaturas. Esta irrupción recurrente de las reformas en la historicidad de los programas de formación docente va reduciendo paulatinamente a las licenciaturas sus posibilidades de afirmarse en un ethos singular de formación docente que pueda contribuir a mejorar la 
calidad de la educación.

\section{Conclusiones}

El análisis de este archivo pone de manifiesto la necesidad de registrar los movimientos de arriba hacia abajo y de abajo hacia arriba que muestran que los procesos de reforma son complejos y no siempre circunscriben obediencia y cambio en las formas de ver y pensar la formación docente. El primer trayecto de la formación inicial docente que aquí destacamos no había atestiguado el efecto de la regulación neoliberal que se evidencia en el segundo. Esta observación permite colegir tensiones entre las aspiraciones de los formadores de maestros en el área del Inglés que reclaman una subjetivad del docente en formación más ligada a la idea del educador y las exigencias normativas que orientan la formación de un docente certificado para enseñar la lengua. Del educador al docente competente en inglés se advierten principios teleológicos equidistantes.

De otro lado, este análisis es una evidencia clara de los desencuentros entre las políticas educativas y las prácticas de los maestros. Éstas han sido un instrumento útil al engranaje neoliberal que, bajo el discurso de la "eficacia y la eficiencia" busca la homogeneización de la profesión docente con modelos altamente fordistas, donde, como señalamos arriba, la autonomía de los programas de formación docente se ve minada por prácticas de estandarización. Dichas prácticas son todavía más nocivas en la profesión del docente inglés puesto que ésta ha sido reducida al manejo proficiente de la lengua en detrimento de los componentes pedagógicos, didácticos, e investigativos. Sin embargo, a pesar de esas formas de control normativo, los maestros, los formadores de maestros, y los maestros en formación siguen encontrando formas de resistencia que se materializan bien sea en pequeños proyectos innovadores en sus salones de clase o en grandes proyectos como la adopción de perspectivas decoloniales en programas de maestrías y doctorados.

\section{Referencias}

ASCUN (octubre de 2019). Se salvan más de 140 licenciaturas y sus miles de estudiantes. https://ascun.org.co/noticias/detalle/se-salvan-mas-de-140-licenciaturas-y-susmiles-de-estudiantes-f06

Ávalos, B. (2014). La formación inicial docente en Chile: Tensiones entre políticas de apoyo y control. Estudios Pedagógicos, (40), 11-28. https://doi.org/10.4067/s071807052014000200002

Ayala Zárate, J, y Álvarez V, J. A. (2005). A Perspective of the Implications of the Common European Framework Implementation in the Colombian Socio-cultural Context. Colombian Applied Linguistics Journal, (7), 7-26. 
http://www.scielo.org.co/scielo.php?script=sci_arttext\&pid=S0123$46412005000100002 \& \operatorname{lng}=e n \&$ tIng=en

Borg, S. (2003). Teacher cognition in language teaching: A review of research on what language teachers think, know, believe, and do. Language Teaching, (36), 81-109.

Cabra Torres, F., \& Marín Díaz, D. L. (2015). Formar para investigar e innovar: tensiones y preguntas sobre la formación inicial de maestros en Colombia. Revista Colombiana De Educación, 1(68), 149.171. https://doi.org/10.17227/01203916.68rce149.171

Calvo G. (2004). La Formación de los docentes en Colombia. Estudio Diagnóstico. Universidad Pedagógica Nacional. Bogotá.

Calvo, G., Rendón, D. y Rojas, L. (2004). Un diagnóstico de la formación docente en Colombia. Revista Colombiana de Educación, (47), 201-217

Cárdenas Ramos, R. (2009). Tendencias globales y locales en la formación de docentes de lenguas extranjeras. Íkala, Revista de Lenguaje y Cultura, 14(22), 71-106.

Cárdenas, M. (2010). Encuentros y desencuentros en la formación de profesores de inglés en Colombia: una mirada a las políticas del Programa Nacional de Bilingüismo. Formação De Professores De Línguas Na América Latina E Transformação Social, (1), $19-44$

Cárdenas-Vergaño, N. (2018). Perspectivas para un estudio sobre bilingüismo en universidades regionales colombianas. Revista Historia de la Educación Latinoamericana, 20(31). https://doi.org/10.19053/01227238.8566

Castañeda Trujillo, J. (2019). Los profesores de inglés (re)clamando por un cambio en el concepto de formación inicial de profesores. Enletawa Journal, 12(1), 62-79. de https://revistas.uptc.edu.co/index.php/enletawa_journal/article/view/10381

Castañeda-Peña, H. (2018). Structuralist, postructuralist and decolonial identity research in English language teaching and learning: A reflection problematizing the field. En Castañeda-Peña, H.; Guerrero, C.; Méndez, P.; Londoño, A.; Dávila, A.; Arias, C.; Lucero, E.; Castañeda, J.; Posada, J.; Samacá, Y. (2018). ELT Local Research Agendas I. UD Editorial.

CEPAL. (1992). Educación y Conocimiento: Eje de la Transformación Productiva con Equidad. Publicación de Las Naciones Unidas.

Consejo de Bogotá. (2005). Acuerdo 364. Por el cual se institucionalizan el Programa Bogotá Bilingüe en Diez Años $y$ se dictan otras disposiciones. https://concejodebogota.gov.co/cbogota/site/artic/20200917/asocfile/2020091716 0426/edici_n_3065_pa_364_369_pd_de_2020.pdf 
Consejo de Bogotá. (2006). Acuerdo 253. Por el cual se institucionaliza el Programa Bogotá Bilingüe $y$ se dictan otras disposiciones. https://xperta.legis.co/visor/legcol/legcol_759920422b40f034e0430a010151f034/co leccion-de-legislacion-colombiana/acuerdo-253-de-2006

Consejo Nacional de Acreditación de Escuelas Normales Superiores (2000). Formación De Maestros. Elementos para el debate. https://www.mineducacion.gov.co/1621/articles-85742_Archivo_pdf1.pdf

Cuenca, R. (2005). La Formación Docente en América Latina y el Caribe: Tensiones, Tendencias y Propuestas. p. 45 - 55. En: Rendón Lara, D. B. y Rojas García, L. I. El Desafío de Formar los Mejores Docentes. Universidad Pedagógica Nacional.

Cummins, J (2011). Teaching for transformation. What does it mean for English Language Arts in a Multilingual School Context? A Journal of the Texas Council of Teachers of English Language Arts, 41 (1), 4-16.

Davini, M. (1995). La formación docente en cuestión: política y pedagogía. Paidós.

De Mejia, A. M. (2006). Bilingual Education in Colombia: Towards a Recognition of Languages, Cultures and Identities. Colombian Applied Linguistics Journal, (8), 152168.

Elbaz, P. (1983). Teacher thinking: a study of practical knowledge. Croom Helm.

Fandiño, Y. (2013). Knowledge Base and EFL Teacher Education Programs: A Colombian Perspective. Íkala, Revista de Lenguaje y Cultura, 18(1), 83-95. http://www.scielo.org.co/scielo.php?script=sci_arttext\&pid=S0123$34322013000100007 \&$ Ing=en\&tlng=en

Fandiño-Parra, Y. (2011). Programas de formación de docentes de inglés centrados en la reflexión. Educación $\quad y \quad$ Educadores, 14(2). http://educacionyeducadores.unisabana.edu.co/index.php/eye/article/view/1922/2 511

Flores-Arévalo, I (2003) ¿Cómo estamos formando a los maestros en América Latina? Lima. Ministerio de Educación, -GTZ. UNESCO.

Flórez Ochoa, R. (1997). Hacia una Pedagogía del conocimiento. Mc Graw Hill.

Freire, Paulo. (2011). La educación como práctica de la libertad (2 ed.). Siglo XXI Editores.

Foucault, M. (1996.). El sujeto y el poder. Revista de Ciencias Sociales, 11(12), 7-19.

Foucault, M. (1969). Arqueología del Saber. México. Siglo XXI. 
Giroux, Henry A. (1987). La formación del profesorado y la ideología del control social. Revista de educación, 1(284), 53-76

González, A. (2003). "Who is educating EFL teachers: A qualitative study of in-service in Colombia?" ÍKALA: Revista de Lenguaje y Cultura, 8 (14), 153-172. http://aprendeenlinea.udea.edu.co/revistas/index.php/ikala/article/view/3183/2947

González, A. y Quinchía, D. (2003). Tomorrow's EFL teacher educators. Colombian Applied Linguistics Journal,

(5),

86-104. http://calj.udistrital.edu.co/pdf_files/App_2003/Art5.pdf

González, A. y Sierra, N. (2005). The professional development of foreign language teacher educators: Another challenge for professional communities. ÍKALA: Revista de $\begin{array}{lllll}\text { Lenguaje } & y & \text { Cultura, } & 10 & \text { (16), }\end{array}$ http://aprendeenlinea.udea.edu.co/revistas/index.php/ikala/article/view/3049/2824

Granados, C. (2015). Formación inicial de docentes de lenguas extranjeras. Nodos y Nudos, 4(39), 59-69. https://doi.org/10.17227/01224328.4356

Guerrero, C. H. (2008). Bilingual Colombia: What does It Mean to Be Bilingual within the Framework of the National Plan of Bilingualism? Profile, 10 (1), 27-45.

Guerrero, C. H. (2009). Language Policies in Colombia: The Inherited Disdain for our Native Languages. HOW Journal, (16), 11-24.

Imbernón, F. (2001). La profesión docente ante los desafíos del presente y del futuro. En Marcelo, C. (Ed.). La función docente (pp. 27-41). Síntesis.

Jurado Vaencia, F. (2016). Hacia la renovación de la formación de los docentes en Colombia: ruta tradicional y ruta polivalente. Pedagogía y Saberes, (45), 11-22.

Johnson, K. (2009). Second language teacher education: A sociocultural perspective. Routledge.

Kubota, R. (2014). Race and language learning in multicultural Canada: Toward critical antiracism. Journal of Multilingual and Multicultural Development, 36(1), 3-12. https://doi.org/10.1080/01434632.2014.892497

Macías, D. (2013). An Exploration of EFL Teachers' Awareness of the Sources of Pedagogical Knowledge in a Teacher Education Program. Profile: Issues in Teachers ' $\begin{array}{lll}\text { Professional Development, 15(2), 99-114 } & \end{array}$ https://revistas.unal.edu.co/index.php/profile/article/view/40172/42046

Madrid, G. (2004). La formación inicial del profesorado de lengua extranjera. Profesorado, revista de currículum y formación del profesorado, 8(1), 7-19. 
Martínez Boom, A. (2016). La formación en las universidades pedagógicas. Un punto de $\begin{array}{llll}\text { encuentro. Nodos } & Y & \text { Nudos, 4(40), } & 115.120 .\end{array}$ https://doi.org/10.17227/01224328.5251

Martínez, M; Calvo, G., Martínez-Boom, A., Soler, C., Prada, M., (2015). Pensar la formación de maestros hoy. Una propuesta desde la experiencia pedagógica. Alcandía Mayor de Bogotá

Méndez, P. (2017). Sujeto maestro en Colombia, luchas y resistencias. Editorial Universidad Santo Tomás.

Méndez, P., Garzón, E., Noriega, R., Rodríguez, F., Osorio, G. (2019). English Teachers' Subjectivities: Contesting and Resisting Must-be Discourses. English Language Teaching; (12), 3, 65-76. https://doi.org/10.5539/elt.v12n3p65

Méndez, P., Garzón, E., Noriega, R., Rodríguez, F., Osorio, G. (2019). English Teacher, subjetividad y enseñanza del inglés en Bogotá. Editorial UD. Bogotá

Ministerio de Educación Nacional (1994). Ley 115 de 1994. https://www.mineducacion.gov.co/1621/articles-85906_archivo_pdf.pdf

Ministerio de Educación Nacional (1996). Decreto 0709 de Abril 17 de 1996. https://www.mineducacion.gov.co/1621/articles-86215_archivo_pdf.pdf

Ministerio de Educación Nacional. (1996). Plan Nacional de Educación, 1996-2005.

https://www.mineducacion.gov.co/1621/articles-85242_archivo_pdf.pdf

Ministerio de Educación Nacional (1998). Decreto 0272 de 1998. https://www.mineducacion.gov.co/1621/articles-86202_archivo_pdf.pdf

Mora, Alberto, Trejo, Paulina and Roux, Ruth (2014). English Language Teachers' Professional Development and Identities. Profile, 16(1), 49-62

Munévar García, P. A. (2019). Capítulo 1. La formación de maestros en Colombia: tendencias, tensiones y prospectiva frente a la resignificación del papel del maestro colombiano. https://doi.org/10.22490/9789586516624.01

Perrenoud, P. (2001). La formación de los docentes en el siglo XXI. Revista de Tecnología Educativa, XIV (3), 503-523.

Pineda, Báez, Clelia, \& Clavijo Olarte, Amparo. (2003). Growing together as teacher researchers. Colombian Applied Linguistics Journal, 1(5), 65-85

Popkewitz, T. (1990). Ideología y formación social en la educación del profesorado. En 
Popkewitz (coord.). Formación del profesorado: tradición, teoría y práctica. Universitat de València, Servei de Publicacions (6-34).

Richards, J. C., \& Lockhart, C. (1994). Reflective teaching in second language classrooms. Cambridge University Press.

Rosa J. \& Flores, N. (2015). Undoing Appropriateness: Raciolinguistic Ideologies and Language Diversity in Education. Harvard Educational Review, 85(2), 149-171.

Rubiano, C. I., Frodden, C. y Cardona, G. (2000). The impact of the Colombian framework for English (COFE) project: an insiders' perspective. Íkala, 5(1-2), 37-54. https://revistas.udea.edu.co/index.php/ikala/article/view/8545

Saldarriaga, O. (2009). De la pedagogía al saber pedagógico. Notas para (un) saber del currículo. Cuadernos de psicopedagogía, (5), 1-23. https://revistas.uptc.edu.co/index.php/psicopedagogia/article/view/527

Shulman, L. (1987). Knowledge and Teaching: Foundations of the New Reform, Harvard Educational Review, 57(1), 1-22.

Tenti, E. (2009). Reflexiones sobre la construcción social del oficio docente. En Vélaz-De Medrano, C. y Vaillant, D. (Coords.) Aprendizaje y desarrollo profesional docente. OEI \& Fundación Santillana

Terigi, F. (2009). Carrera Docente y políticas de desarrollo profesional. En Vélaz-De Medrano, C. y Vaillant, D. (Coords.) Aprendizaje y desarrollo profesional docente. OEI \& Fundación Santillana

Tezanos, A. (1982). Notas para una reflexión crítica sobre la Pedagogía. En Hoyos, G. (ed.), EI Sujeto como objeto en las Ciencias Sociales. CINEP.

Torres, R. M. (1996). Formación docente: Clave de la reforma educativa. En Nuevas formas de enseñar y de aprender. Demandas a la educación inicial de los educadores, Santiago. UNESCO-OREALC.

UNESCO. (1990). Satisfacción de las necesidades Básicas de Aprendizaje. Jomtien.

UNESCO. (1991). Informe final. Cuarta Reunión del Comité Regional Intergubernamental del Proyecto Principal. De la Esfera de la Educación en América Latina y El Caribe.

UNESCO-OREALC. (1995). Jornadas de reflexión pedagógica: formación docente inicial para los profesores de la educación básica. UNESCOOREALC.

Vaillant, D. (2009). Políticas para un desarrollo profesional docente efectivo. En Vélaz-De Medrano, C. y Vaillant, D. (coords). Aprendizaje y Desarrollo Profesional Docente. 
Metas Educativas 202 (pp. 29-38). Fundación Santillana.

Vaillant, D. (2005). Formación de docentes en América Latina. Re-inventado el modelo tradiciona/Barcelona, Editorial Octaedro

Vaillant, D. (2010a). Capacidades docentes para la educación del mañana. Pensamiento Iberoamericano, 7, 113-128

Valencia, S. (2005). Bilingualism and English language teaching in Colombia: A critical Outlook. ELT.

Vasco, C, Vasco, E, Martínez, A. (2008) Educación, Pedagogía y Didáctica: Una perspectiva epistemológica. En: Hoyos, G. (E). Enciclopedia Iberoamericana de Filosofía: Filosofía de la Educación. Editorial Trotta

Zuluaga, O.L. (1999). Pedagogía e Historia: la historicidad de la pedagogía, la enseñanza, un objeto de saber. Siglo del Hombre Editores

\section{Agradecimientos y ayuda financiera:}

Este artículo de investigación es resultado de la relación normas-formación docente resultado de la investigación "English Language Teachery enseñanza del Inglés en Bogotá 2004-2015 (código 4-165-529-16) financiado por el CIDC de la Universidad Distrital Francisco José de Caldas. Bogotá-Colombia. 
Anexo 1: Eje normativo

\begin{tabular}{|c|c|c|c|c|c|c|}
\hline $\begin{array}{l}\text { Ley } 30 \mathrm{de} \\
1992\end{array}$ & $\begin{array}{l}\text { Ley } 115 \text { de } \\
1994\end{array}$ & $\begin{array}{l}\text { Decreto } \\
0709 \\
1996\end{array}$ de & \begin{tabular}{|l|} 
Decreto \\
3012 \\
1997
\end{tabular} de & $\begin{array}{l}\text { Plan } \\
\text { Decenal } \\
\text { 1996-2005 }\end{array}$ & $\begin{array}{l}\text { Decreto } 0272 \text { de } \\
1998\end{array}$ & $\begin{array}{l}\text { Res. } 2041 \mathrm{de} \\
\text { febrero de } \\
2016 \text { ) }\end{array}$ \\
\hline 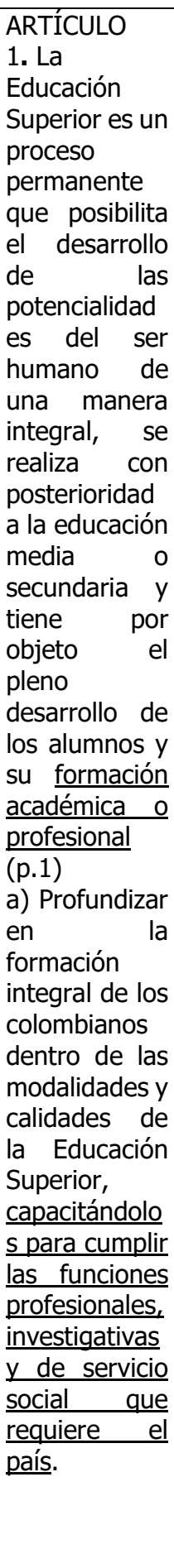 & 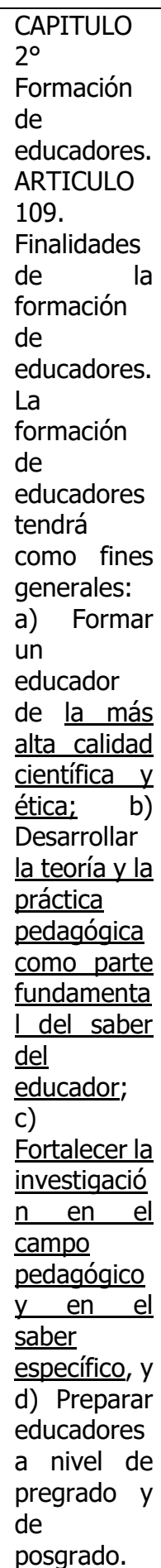 & 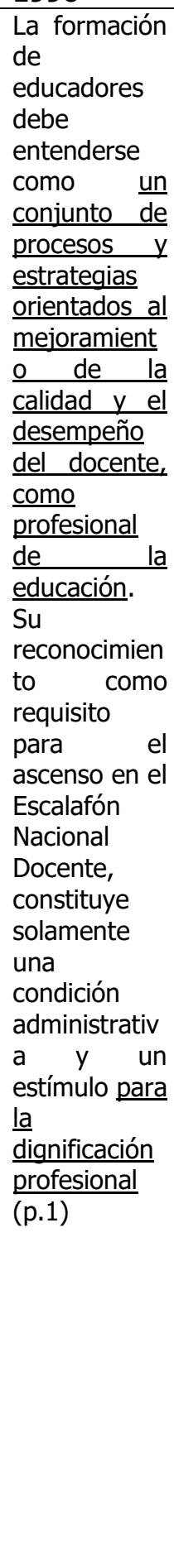 & $\begin{array}{l}\text { Que de } \\
\text { acuerdo con } \\
\text { lo dispuesto } \\
\text { por el artículo } \\
112 \text { de la Ley } \\
115 \text { de 1994, } \\
\text { la formación } \\
\text { profesional } \\
\text { de les } \\
\text { educadores } \\
\text { corresponde } \\
\text { a las } \\
\text { universidades } \\
\text { y a las demás } \\
\text { instituciones } \\
\text { de educación } \\
\text { superior que } \\
\text { posean una } \\
\text { facultad de } \\
\text { educación u } \\
\text { otra unidad } \\
\text { académica } \\
\text { dedicada a la } \\
\text { educación y a } \\
\text { las escuelas } \\
\text { normales } \\
\text { debidamente } \\
\text { reestructurad } \\
\text { as } \\
\text { aprobadas y } \\
\text { como } \\
\text { escuelas } \\
\text { normales } \\
\text { superiores, } \\
\text { para atender } \\
\text { programas de } \\
\text { formación de } \\
\text { docentes } \\
\text { para el nivel } \\
\text { de preescolar } \\
\text { y el ciclo de } \\
\text { básica } \\
\text { primaria. }\end{array}$ & $\begin{array}{l}\text { Y en esta } \\
\text { propuesta } \\
\text { consideramos } \\
\text { que la } \\
\text { Universidad un } \\
\text { juega un } \\
\text { papel } \\
\text { decisivo, } \\
\text { porque hoy en } \\
\text { día es } \\
\text { absolutament } \\
\text { e claro, para la } \\
\text { mayoría de la } \\
\text { población, que } \\
\text { el sistema } \\
\text { educativo no } \\
\text { solo culmina } \\
\text { sino que } \\
\text { también } \\
\text { empieza con } \\
\text { la la la } \\
\text { Universidad, } \\
\text { ya que la } \\
\text { misma } \\
\text { formación del } \\
\text { hogar, desde } \\
\text { la lanificación } \\
\text { planion } \\
\text { familiar y la } \\
\text { educación } \\
\text { prenatal del } \\
\text { futuro niño, } \\
\text { son materias } \\
\text { universitarias } \\
\text { o asunto de la } \\
\text { universidad. } \\
\text { Así como lo } \\
\text { es, la la } \\
\text { igualmente, la } \\
\text { tarea de la } \\
\text { formación del } \\
\text { educador que } \\
\text { lleva encima la } \\
\text { mayor carga } \\
\text { de responsabilida } \\
\text { d, el maestro } \\
\text { o maestra } \\
\text { preescolar } \\
\text { (p.2) }\end{array}$ & 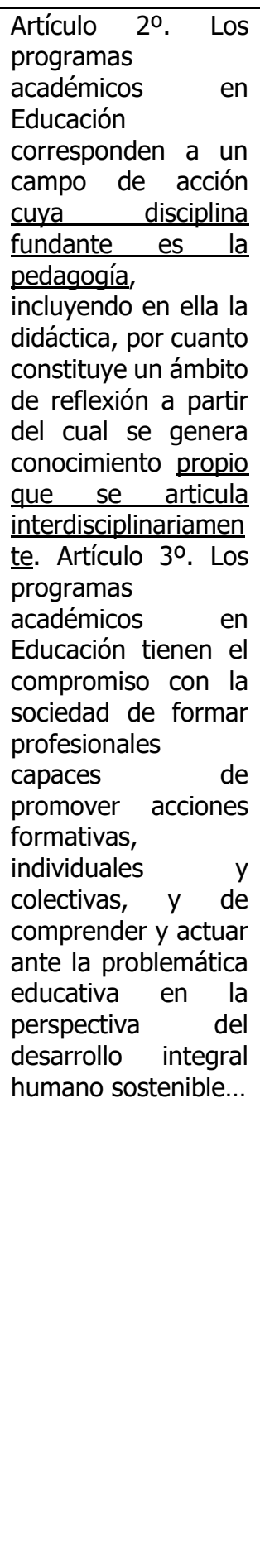 & $\begin{array}{l}\text { Que en las } \\
\text { bases del Plan } \\
\text { Nacional de } \\
\text { Desarrollo } \\
2014-2018, \\
\text { se reconoció la } \\
\text { importancia de } \\
\text { la oferta y el } \\
\text { desarrollo de } \\
\text { los programas } \\
\text { académicos } \\
\text { pertenecientes } \\
\text { al área del } \\
\text { conocimiento } \\
\text { del campo de } \\
\text { la Educación, } \\
\text { contemplando } \\
\text { la necesidad } \\
\text { de una política } \\
\text { de } \\
\text { mejoramiento } \\
\text { del sistema } \\
\text { educativo del } \\
\text { país, proceso } \\
\text { que inicia } \\
\text { desde la } \\
\text { adecuación de } \\
\text { las condiciones } \\
\text { en la } \\
\text { formación de } \\
\text { quien se } \\
\text { prepara } \\
\text { profesionalmen } \\
\text { te para el } \\
\text { ejercicio } \\
\text { docente, con la } \\
\text { premisa de que } \\
\text { la excelencia } \\
\text { de los } \\
\text { educadores es } \\
\text { un factor } \\
\text { esencial para } \\
\text { garantizar la } \\
\text { calidad de la } \\
\text { educación. }\end{array}$ \\
\hline
\end{tabular}

